\title{
Literatur als adelige Hausüberlieferung?
}

\author{
KLAUS GraF (Koblenz)
}

In Achim von Arnims historischem Roman 'Die Kronenwächter, einem phantastischen, heute kaum noch lesbaren Werk, das 1817 im Druck erschien, ist die achte Geschichte des zweiten Buches überschrieben: Das Hausmärchen. Berthold, der Held des Buches, das im frühen 16. Jahrhundert spielt, ist ein Abkömmling der Hohenstaufen. Eines Tages erhält er Besuch von dem Ritter Ehrenhalt, einem Gesandten der Kronenwächter, die als eine Art Gralsritter die Krone der Hohenstaufen bewachen. Der Ehrenhalt bringt Berthold eine Bilderhandschrift mit, in der von Bertholds Abstammung erzählt wird. In ihren einsamen Wachstunden unterhalten sich die Kronenwächter, erzählt der Bote, bald mit den wohlbezeugten Geschichten des staufischen Hauses, bald mit Hausmärchen aus den Zeiten des Attila, von denen eines in dem Buch abgebildet sei. Arnim erspart es dem Leser leider nicht, dieses Hausmärchen, in dem bezeichnenderweise ein Graf der Nibelungen am Bodensee auftritt, in ganzer Länge zur Kenntnis zu nehmen.' Hier ist es zwar durchaus entbehrlich, doch mag es vielleicht von Nutzen sein, für das weitere im Gedächtnis zu behalten, daß die Rede von heroischer Tradition als adeliger Hausüberlieferung auch eine romantische Fiktion sein kann.

In seiner Einführung `Das Nibelungenlied` hat Joachim Heinzle die Auffassung vertreten, die Heldensage sei zu einem guten Teil als Familientradition oder >Hausüberlieferung herrschender Geschlechter tradiert worden. ${ }^{2} \mathrm{Er}$ weist zwar darauf hin, daß es keine direkten Zeugnisse für die Pflege der Nibelungensage als Hausüberlieferung aus der Zeit vor der Verschriftlichung des Stoffes gebe, macht jedoch gleichzeitig auf die Vermutung aufmerksam, $\mathrm{da} ß$ es in "verschiedenen vornehmen Familien Tradition gewesen ist, Namen aus der Heldensage zu führen« (S. 30). Wenn es gelänge, die beträchtlichen methodischen und sachlichen Probleme dieses neuen Zugangs zur Heldensage zu bewältigen, eröffne sich, »die faszinierende Aussicht, die Verbreitung der Sage im Reflex der Namenbelege zeitlich und räumlich genau zu fixieren« (S. 30).

1 A. von Arnim, Werke, Bd. 2: Die Kronenwächter, hrsg. v. P.M. Lützeler, Frankfurt a. M. 1989 (Bibliothek deutscher Klassiker 42), S. 220-229. Arnim selbst hielt das Hausmärchen für den »Mittelpunkt« seines Romans (ebd., S. 642).

2 J. Heinzle, Das Nibelungenlied. Eine Einführung, München und Zürich 1987 (Artemis Einführungen 35), S. 29. 
Es waren vor allem zwei Historiker, Reinhard Wenskus und Wilhelm Störmer, die in den 70er Jahren den Personennamensbestand des Nibelungenliedes mit von ihnen erschlossenen frühmittelalterlichen Adelsgruppen des 8 . und 9. Jahrhunderts in Verbindung gebracht haben. 1973 erschien sowohl Wenskus' Aufsatz , Wie die Nibelungenüberlieferung nach Bayern kam ${ }^{3}$ als auch Störmers Habilitationsschrift mit dem programmatischen Titel $\gg$ Früher Adek, in der die Vergabe von Personennamen aus dem Nibelungenlied als "ein archaischer Akt der >Ansippung ‘ an die heldenhaften Leitbilder zur Erhöhung des aristokratischen Prestiges « vorgestellt wird. ${ }^{4}$ Störmer hat seine Auffassung auch in zwei Aufsätzen aus den Jahren 1974 und $1977^{5}$ und zuletzt in dem 1987 erschienenen Tagungsband der Passauer Nibelungengespräche von 1985 bekräftigt. ${ }^{6}$ Eine intensive Auseinandersetzung mit den Thesen beider Forscher hat für das Nibelungenlied, soweit mir bekannt, weder unter Historikern noch unter Germanisten stattgefunden. Lediglich für den von Wenskus behandelten Iring-Stoff liegt eine Stellungnahme von Hilkert Weddige vor. ${ }^{7}$ Obwohl sich Weddige intensiv mit den historischen Trägern der Namen Iring und Irminfried auseinandergesetzt hat, kommt er zu dem Schluß, von einer »Interdependenz zwischen Iringsage und adeliger Hausüberlieferung« sei »nichts zu entdecken« (S. 75). Und weiter: »Von historischer Seite ist eine solche im Falle der Nibelungensage allzu selbstverständlich unterstellt worden« (ebd.)

Der Historiker, der sich daran macht, die Behauptungen über den $\mathrm{Zu}$ sammenhang zwischen Heldensagen und sadeliger Hausüberlieferung kritisch zu überprüfen, kommt nicht darum herum, sich mit den methodischen und sachlichen Prämissen zu beschäftigen, die der Hauckschen Kategorie der radeligen Hausüberlieferung` und dem von Wenskus und Störmer vertretenen

3 R. Wenskus, Wie die Nibelungen-Überlieferung nach Bayern kam, Zeitschrift für bayerische Landesgeschichte 36 (1973), S. 393-449; übernommen als Exkurs I in: ders., Sächsischer Stammesadel und fränkischer Reichsadel, Göttingen 1976 (Abhandlungen der Akademie der Wissenschaften in Göttingen. Philol.-Hist. K1. 3. Folge 93), S. 477-529 (hiernach zitiert).

4 W. Störmer, Früher Adel. Studien zur politischen Führungsschicht im fränkischdeutschen Reich vom 8. bis 11. Jahrhundert, Stuttgart 1973 (Monographien zur Geschichte des Mittelalters 6, I-II), Bd. 2, S. 497. Vgl. die eingehende Besprechung von F. Graus, Schweizerische Zeitschrift für Geschichte 24 (1974), S. 294-298.

5 W. Störmer, Die Herkunft Bischof Pilgrims von Passau (971-991) und die Nibelungen-Überlieferung, Ostbairische Grenzmarken 16 (1974), S. 62-67; ders., Adel und Ministerialität im Spiegel der bayerischen Namengebung (bis zum 13. Jahrhundert). Ein Beitrag zum Selbstverständnis der Führungsgruppen, DA 33 (1977), S. 84-152, hier S. 101-112.

6 W. Störmer, Nibelungentradition als Hausüberlieferung in frühmittelalterlichen Adelsfamilien? Beobachtungen zu Nibelungennamen im 8./9. Jahrhundert vornehmlich in Bayern, in: F.P. Knapp (Hrsg.), Nibelungenlied und Klage. Sage und Geschichte, Struktur und Gattung. Passauer Nibelungengespräche, Heidelberg 1987, S. $1-20$.

$7 \mathrm{H}$. Weddige, Heldensage und Stammessage. Iring und der Untergang des Thüringerreiches in Historiographie und heroischer Dichtung, Tübingen 1989 (Hermaea NF 61); vgl. auch meine Rezension, GRM 72 (1991), S. 101-103. 
Zweig der Adelsforschung zugrunde liegen. Mein erster Kritikpunkt gilt zugleich der Periodisierung. Früh- und hochmittelalterlicher Adel sind nicht dasselbe. Es geht nicht an, mit spätmittelalterlichen Formen genealogischen Interesses zu argumentieren, da die zugrunde liegende Kontinuitätsprämisse alles andere als gesichert ist. Die Fee Melusine, die angebliche Ahnfrau einer französischen Adelsfamilie im 14. und 15. Jahrhundert, muß also aus dem Spiel bleiben. Vor allem Karl Schmid, der wohl bekannteste Vertreter der von Gerd Tellenbach initiierten Adelsforschung, hat immer wieder auf die Bedeutung des 11. Jahrhunderts für die Formierung des Adels und das adelige Selbstverständnis hingewiesen. Erst im 11. Jahrhundert sind >Adelshäuser< identifizierbar, da sie sich nun nach einem >Hausı, meist einer Höhenburg, nennen. Um die Mitte des 11. Jahrhunderts verlassen die Grafenfamilien die dörflichen Herrenhöfe und ziehen auf Burgen. Erst im 12. Jahrhundert entstehen in Deutschland dann die hausgeschichtlich orientierten Quellen, etwa im Umkreis der Welfen. ${ }^{8}$ Das ist alles nicht neu, vermag jedoch meine erste These plausibel zu machen:

1. Wer sowohl frühmittelalterliche als auch hoch-oder gar spätmittelalterliche Zeugnisse einem einheitlichen Begriff von sadeliger Hausüberlieferung subsumiert, verkennt den entscheidenden Wandel des adeligen Selbstverständnisses.

Karl Schmid und sein Schüler Gerd Althoff haben sich eingehend mit den Texten beschäftigt, die man als Spiegel radeliger Hausüberlieferung in Anspruch genommen hat. Bereits 1964 fand Schmid die von Karl Hauck in bezug auf die >Translatio sancti Alexandri< und Widukinds Sachsengeschichte gewählte Bezeichnung `Hausüberlieferung »nicht glücklich und treffend«, da das Herkunfts-, Stammes- und Sippenbewußtsein dieser Quellen eben nicht eine besondere Form (etwa die igenealogia $)$ finde. Als charakteristisches Beispiel für das Genus `Hausüberlieferung nannte Schmid die beiden Texte der 'Genealogia und 'Historia Welforum aus dem 12. Jahrhundert. ${ }^{9}$ Gerd Althoff hat 1988 angemerkt, der Begriff `Hausüberlieferung sei nicht so »unumstritten wie man angesichts der häufigen Verwendung in der Literatur meinen könnte« ${ }^{10}$ Wiederholt hat Althoff die pointierte These vertreten, daß Texte, die man der adeligen Hausüberlieferung zugerechnet hat, in Wirklichkeit die Interessen der durch Krisen bedrängten geistlichen Gemeinschaft, in der sie

8 Es genüge der Hinweis) auf J. Bumke, Höfische Kultur. Literatur und Gesellschaft im hohen Mittelalter, Bd. 2, Stuttgart 1986, S. 617-621.

9 K. Schmid, Gebetsgedenken und adliges Selbstverständnis im Mittelalter. Ausgewählte Beiträge, Sigmaringen 1983, S. 82, Anm. 88.

10 G. Althoff, Causa scribendi und Darstellungsabsicht: Die Lebensbeschreibungen der Königin Mathilde und andere Beispiele, in: Litterae medii aevi, FS J. Autenrieth, Sigmaringen 1988, S. 117-133, hier S. 120; vgl. auch ders., Niedersächsisches Jahrbuch für Landesgeschichte 60 (1988), S. 318; E. Karpf, Herrscherlegitimation und Reichsbegriff in der ottonischen Geschichtsschreibung des 10. Jahrhunderts, Stuttgart 1985 (Historische Forschungen 10), S. 188-190. 
entstanden sind, oder gar konkurrierender Adelsfamilien wiedergeben." >Adelige Hausüberlieferung und vielfach tendenziöse >Aussagen über adelige Häuser sind eben nicht dasselbe. Eine genealogische Aufzeichnung, die im Zuge eines Erbschaftsprozesses aufgrund zahlreicher Zeugenaussagen und unter Zuhilfenahme schriftlicher Quellen abgefaßt wurde, hat einen anderen Stellenwert als ein >genealogisches Herkommen', das eine fiktive Herkunft des Geschlechts behauptet, also eine sogenannte `Geschlechtersage $<$. Will man die Eigen- und die Fremdsicht einer Adelsfamilie nicht einebnen, so muß man eine >Autorisierung der entsprechenden Hausüberlieferung durch das Geschlecht selbst belegen können. Außerdem sollte eine Hausüberlieferung tatsächlich mehr als eine Generation als Überlieferung faßbar sein. Bei Vorstellungen und Meinungen, die nur punktuell, in einem einzigen Zeugnis greifbar sind, ist also Vorsicht geboten. Meine zweite These lautet:

2. Als sadelige Hausüberlieferung sollte eine Tradition nur dann bezeichnet werden, wenn Anhaltspunkte dafür vorliegen, daß die entsprechende Überlieferung von dem betreffenden Adelshaus auch akzeptiert wurde.

Beispielsweise gibt es keinerlei hinreichende Indizien dafür, daß der im 12. Jahrhundert entstandene >König Rother tatsächlich, wie Störmer will, von dem Adelshaus der Tenglinger selbst als »Propagierung der eigenen >Haus-Geschichte im Rahmen der Reichsgeschichter verstanden wurde. ${ }^{12}$

Liest man Haucks erstmals 1954 publizierten Aufsatz >Haus- und sippengebundene Literatur mittelalterlicher Adelsgeschlechter von Adelssatiren des 11. und 12. Jahrhunderts her erläutert ${ }^{13}$, so stößt man wiederholt auf Stellen, in denen Hauck von der Existenz einer in vorchristliche Zeiten zurückgehen-

11 Vgl. zuletzt zusammenfassend G. Althoff, Verwandte, Freunde und Getreue. Zum politischen Stellenwert der Gruppenbindungen im früheren Mittelalter, Darmstadt 1990, S. 67-77. Vgl. ebd., S. 76f.: »Wir besitzen keine unmittelbaren Selbstzeugnisse, sondern heterogene Aufzeichnungen, deren Funktion und Intention zu klären ist, bevor man aus ihnen auf die Selbstsicht der Adelsgeschlechter schließt«. Vgl. auch ders., Genealogische Fiktionen und die historiographische Gattung der Genealogie im hohen Mittelalter, in: Staaten, Wappen, Dynastien, Innsbruck 1988 (Veröffentlichungen des Stadtarchivs Innsbruck NF 18), S. 67-79.

12 W. Störmer `Spielmannsdichtung und Geschichte. Die Beispiele $>$ Herzog Ernst‘ und ২König Rother`, Zeitschrift für bayerische Landesgeschichte 43 (1980), S. 551-574, hier S. 564f. Ähnlich auch U. Meves, Zur Rolle der Sieghardinger für die Adelsliteratur im Südosten des Reiches $(10 .-13$. Jh.). Auf der Grundlage personen- und besitzgeschichtlicher Überlegungen, in: $\mathrm{H}$. Wenzel (Hrsg.), Adelsherrschaft und Literatur, Bern 1980 (Beiträge zur älteren deutschen Literaturgeschichte 6), S. 115-180, hier S. 138. Zu Recht zurückhaltend dagegen J. Bumke, Mäzene im Mittelalter. Die Gönner und Auftraggeber der höfischen Literatur in Deutschland 1150-1300, München 1979, S, 91-94. Auch bei spätmittelalterlichen literarischen Texten, die von der Forschung auf das genealogische Interesse einer Adelsfamilie zurückgeführt wurden, sind begründete Zweifel an dieser Hypothese angebracht, vgl. etwa K. Graf, Genealogisches Herkommen bei Konrad von Würzburg und im ,Frieden von Schwaben`, Jahrbuch der Oswald von Wolkenstein Gesellschaft 5 (1988/1989), S. 285-295.

13 K. Hauck, Haus- und sippengebundene Literatur mittelalterlicher Adelsgeschlechter von Adelssatiren des 11. und 12. Jahrhunderts her erläutert: in: W. Lammers (Hrsg.), Geschichtsdenken und Geschichtsbild im Mittelalter, Darmstadt 1961 (WdF 21), S. 165-199. 
den, ursprünglich sakralen Haustradition des Adels ausgeht. Seine Kontinuitätsprämissen und seine Betonung des Adelsheils sind von dem Paradigma der 'germanischen Adelsherrschaftı nicht zu lösen. Die ideologischen Prämissen dieses Ansatzes sind etwa von František Graus deutlich hervorgehoben worden. ${ }^{14}$ Einen der Hintergründe der Bemühungen, nibelungische Haustradition auszumachen, fasse ich mit meiner dritten These:

3. Das von Karl Hauck vertretene Interpretament sadelige Hausüberlieferung` ist eng mit einer von der neueren Forschung weitgehend abgelehnten charismatisch akzentuierten Adelskonzeption verknüpft.

Von der Forschung rezipiert wurde denn auch weniger diese Konzeption als das griffige Schlagwort `adelige Hausüberlieferung^ und die vage Vorstellung, daß es sich dabei um etwas bedeutsames handelt. Als Inhalt der Hausüberlieferung präsentierte Hauck vor allem die sprimodiale Tat‘ des 'Spitzenahnsく. Diese ressentialistische< Festlegung, was Hausüberlieferung eigentlich sei, verkennt jedoch das reiche inhaltliche Spektrum und die Perspektivität der in den Quellen bezeugten Familienüberlieferungen. Was berechtigt zu der Annahme, die Krieger und nicht etwa die Geistlichen oder die Frauen einer Familie dürften als die entscheidenden Träger der Tradition gelten? In den familiengeschichtlichen Aufzeichnungen Hermanns des Lahmen aus dem 11. Jahrhundert »lief die Geschichte seiner Vorfahren auf zölibatär lebende Mönche und nicht auf eine blühende Adelsherrschaft zu ${ }^{15}$ Vermutlich hatte er sein genealogisches Wissen von seiner Mutter. ${ }^{16}$ Literarisch tätige Frauen des ottonischen Hauses entwickelten eine spezifische Sicht ihrer Familiengeschichte, die sie, modern gesprochen, als $>$ Frauengeschichter konzipierten. ${ }^{17}$ Von der Vorstellung einer einheitlichen radeligen Hausüberlieferung gilt es somit Abschied zu nehmen. ${ }^{18}$

14 F. Graus, Verfassungsgeschichte des Mittelalters, HZ 243 (1986), S. 529-589, hier S. 559-565; vgl. auch J.B. Freed, Reflections on the Medieval German Nobility, American Historical Review 91 (1986), S. 553-575. Am Beispiel des Begriffs , Geblütsheiligkeit` zeigt K. Schreiner, Die Wissenschaft von der Geschichte des Mittelalters nach 1945. Kontinuitäten und Diskontinuitäten der Mittelalterforschung im geteilten Deutschland, in: E. Schulin (Hrsg.), Deutsche Geschichtswissenschaft nach dem Zweiten Weltkrieg, München 1989 (Schriften des Historischen Kollegs. Kolloquien 14), S. 87-146, hier S. 128f. den ideologischen Hintergrund unmißverständlich auf.

$15 \mathrm{M}$. Borgolte, Über die persönlichen und familiengeschichtlichen Aufzeichnungen Hermanns des Lahmen. ZGOR 127 (1979), S. 1-15, hier S. 14.

$16 \mathrm{Vgl}$. dazu auch A. Borst, Barbaren, Ketzer und Artisten. Welten des Mittelalters, München 1988, S. 137 mit der bemerkenswerten Feststellung: "Die uns vertraute Traditionspflege in adligen Familien war zu Beginn des elften Jahrhunderts noch schwach entwickelt

17 Vgl. K. Sonnleitner, Selbstbewußtsein und Selbstverständnis der ottonischen Frauen im Spiegel der Historiographie des 10. Jahrhunderts, in: Geschichte und ihre Quellen, FS F. Hausmann, Graz 1987, S. 111-119.

$18 \mathrm{Da}$ in Zeugnissen des Familienbewußtseins die Auswahl der Ahnen und Verwandten stark subjektiv geprägt war und von Fall zu Fall wechseln konnte, betont H. Fichtenau, Lebensordnungen des 10. Jahrhunderts. Studien über Denkart und Existenz im einstigen Karolingerreich, Stuttgart 1984 (Monographien zur Geschichte des Mittelalters 30, I-II), Bd. 1, S. 122, 200. 
Sowohl Wenskus als auch Störmer operieren mit stammesübergreifenden Adelsgruppen, die aus Personenidentifizierungen in Verbindung mit besitzgeschichtlichen Beobachtungen erschlossen wurden. Ich kann hier keine eingehende Kritik dieser genealogisch-besitzgeschichtlichen Methode vorlegen ${ }^{19}$, sondern verweise auf den empirischen Teil meiner Ausführungen, aus dem die methodischen Schwachstellen deutlich genug hervorgehen dürften. 1967 schrieb Karl Schmid: »Die Forschung verfügt nicht über eine zureichende Methode, die in der frühmittelalterlichen Überlieferung vorkommenden Personen wenigstens zu einem guten Teil schlüssig identifizieren zu können. Solange das nicht gelingt, wird auch kaum der Personenkreis klar festzustellen und einzugrenzen sein, der zur Führungsschicht zu rechnen ist $\ll .^{20}$ Ein zweites Zitat von Hans K. Schulze aus dem Jahr 1978 mag die Problematik des frühmittelalterlichen Adelsbegriffs weiter verdeutlichen: "Letztlich arbeitet die Adelsforschung mit dem Axiom, daß alle Personen, die nicht ausdrücklich als Unfreie oder Minderfreie bezeichnet werden, dem Adel angehört haben. Da es in der Tat keine Möglichkeit gibt, die Tradenten, die Zeugen der Urkunden, die Insassen der Klöster und die Personen, deren Namen in den Gedenkbüchern verzeichnet sind, in adlige und nichtadlige zu scheiden, kommt man mit einem unspezifischen Adelsbegriff in große Schwierigkeiten. $\ll^{21}$ Die Konsequenz daraus zieht meine These:

4. Nur mit großen Vorbehalten kann von einem frühmittelalterlichen 'Adek gesprochen werden, zumal das von der besitzgeschichtlich-genealogischen Forschung suggerierte Bild weiträumig agierender Adelsverbände aufgrund der nicht behebbaren Schwierigkeiten der Personenidentifizierung methodisch fragwürdig ist.

Wege und Irrwege der westdeutschen `Adelsforschung können hier nicht nachgezeichnet werden. Ausgeklammert werden im folgenden die verfassungsgeschichtlichen ${ }^{22}$ und die begriffsgeschichtlichen ${ }^{23}$ Aspekte. Welche Zir-

19 F. Prinz, Frühes Mönchtum im Frankenreich. Kultur und Gesellschaft in Gallien, den Rheinlanden und Bayern am Beispiel der monastischen Entwicklung (4. bis 8. Jahrhundert), 2. Aufl., Darmstadt 1988, S. 638, erscheint die Frage berechtigt, "ob es sich eigentlich gelohnt hat, mit einer schwer nachzukontrollierenden Subtilität und bei den immer noch beträchtlichen und unvermeidlichen Fehlerquellen der Namensidentifikation eine Fallstudie dieser Art durchzuführen. Jedenfalls zeichnen sich hier absolute Grenzen der von Josef Sturm entwickelten und von der Tellenbach-Schule weiträumig angewandten besitzgeschichtlich-genealogischen Methode ab«. Vgl. auch ebd., S. 642 Anm, 26. Weitere Nachweise von kritischen Stellungnahmen in meiner Rezension des Sammelbandes ,Früh- und hochmittelalterlicher Adel in Schwaben und Bayern<, Zeitschrift für Hohenzollerische Geschichte 24/25 (1988/89), S. 241-243.

20 Schmid [Anm. 9], S. 370.

21 H.K. Schulze, Reichsaristokratie, Stammesadel und fränkische Freiheit. Neuere Forschungen zur frühmittelalterlichen Sozialgeschichte, HZ 227 (1978), S. $353-373$, hier S. 373 .

$22 \mathrm{Vgl}$. Th. Zotz, Grafschaftsverfassung und Personengeschichte. Zu einem neuen Werk über das karolingerzeitliche Alemannien, ZGOR 136(1988), S. 1-16; J. Weitzel, Dinggenossenschaft und Recht. Untersuchungen zum Rechtsverständnis im fränkisch-deutschen Mittelalter, Köln-Wien 1985 (Quellen und Forschungen zur 
kelschlüsse die archäologische Forschung durch die Übernahme vermeintlich gesicherter historischer Ergebnisse über den frühmittelalterlichen >Adek belastet haben, ist von Heiko Steuer nicht zuletzt am Beispiel der sogenannten >Adelsgräber ausführlich dargelegt worden. ${ }^{24}$ Hier kann nur die Frage gestellt, nicht jedoch beantwortet werden, ob der geschichtswissenschaftliche Paradigmenwechsel nicht auch eine Revision des gängigen Bilds von der laikalen >Adelsliteratur des frühen Mittelalters nötig macht. ${ }^{25}$

Den Schluß der methodischen Vorüberlegungen bildet die Frage, welche methodischen Kriterien man anlegen muß, um einen Zufallsbefund von aussagekräftigen Zeugnissen zu scheiden. ${ }^{26}$ Die Diskussion der germanistischen Forschung hat ergeben, $\mathrm{da} \mathrm{B}$ isolierte Personennamen aus der Heldensage nicht als Indiz für die Kenntnis der literarischen Stoffe verwertet werden dürfen. ${ }^{27}$ Ich stimme Störmer völlig zu, wenn er 1987 resümierend betont:

höchsten Gerichtsbarkeit im Alten Reich 15), bes. Bd. 1, S. 138. Durchaus ernst zu nehmen ist der umfangreichste Angriff auf das im Gefolge Heinrich Dannenbauers entwickelte Paradigma, den Wilhelm Schneider führte, vgl. W. Schneider, Arbeiten zur alamannischen Frühgeschichte, Heft IX: Wider die These von der Adelsherrschaft, Tübingen 1988; vgl. auch die weiteren Hefte, etwa Heft XV: Arbeiten zur Agrargeschichte Teil 3, Tübingen 1988, S. 107-116.

23 Vg. H.-W. Goetz, »Nobilis«. Der Adel im Selbstverständnis der Karolingerzeit, Vierteljahrschrift für Sozial- und Wirtschaftsgeschichte 70 (1983), S. 153-191; Fichtenau [Anm. 18], Bd. 1, S. 185-215; O.G. Oexle, Aspekte der Geschichte des Adels im Mittelalter und in der Frühen Neuzeit, in: H.-U. Wehler (Hrsg.), Europäischer Adel 1750-1900, Göttingen 1990 (Geschichte und Gesellschaft. Sonderheft 13), S. $19-56$.

$24 \mathrm{H}$. Steuer, Frühgeschichtliche Sozialstrukturen in Mitteleuropa. Eine Analyse der Auswertungsmethoden des archäologischen Quellenmaterials, Göttingen 1982 (Abhandlungen der Akademie der Wissenschaften in Göttingen. Philol.-Hist. K1. 3. Folge 128). Vgl. dazu auch die kritischen Zitate bei Schneider [Anm. 22], Heft X: Arbeiten zur Archäologie Teil 1, Tübingen 1982, S. 211-239.

$25 \mathrm{Vgl}$. zusammenfassend W. Haubrichs, Geschichte der deutschen Literatur von den Anfängen bis zum Beginn der Neuzeit. Bd. I: Von den Anfängen bis zum hohen Mittelalter. Teil 1: Die Anfänge: Versuche volkssprachiger Schriftlichkeit im frühen Mittelalter (ca. 700-1050/60), Frankfurt a.M. 1988, passim.

26 Die Wahrscheinlichkeit von zufälligen Übereinstimmungen wird von der personengeschichtlichen Forschung im allgemeinen erheblich zu gering veranschlagt. Folgendes stark simplifizierendes Modell möge diese Behauptung belegen: Im Huosigau sollen genau 100 verschiedene Namen mit gleicher Häufigkeit gebräuchlich sein. Wählt man nun zwei Gruppen (Zeugenlisten) von je 10 Personen zufällig aus, so ist die Wahrscheinlichkeit, daß zwei oder mehr Namen in beiden Gruppen vertreten sind, mit etwa 26 Prozent relativ hoch. Wenn aber sowohl in einem Dorf am Mittelrhein als auch in einem Dorf bei Freising ein bestimmtes Namenspaar begegnet, wird dieser Befund von der genealogischen-besitzgeschichtlichen Forschung kritiklos als Beweis der Personenidentität beansprucht. Für recht aufwendige Berechnungen bin ich Herrn Diplom-Volkswirt Dr.U. Scheurle, Stuttgart-Hohenheim, zu Dank verpflichtet.

27 Vgl. zuletzt Weddige [Anm. 7], S. 69, 72; H. Rosenfeld, Ausgewählte Aufsätze zur deutschen Heldendichtung und zur Namenforschung, zur Todes- und Totentanzdichtung, zum Volksdrama und zur Wechselwirkung von Kunst und Dichtung im Mittelalter, hrsg. v. H.-A. Klein, Göppingen 1987 (GAG 473), S. 139-174. 
„Personennamen des Nibelungensagenstoffes, die in Quellen des 8./9. Jahrhunderts erscheinen, vermögen, wenn sie einzeln und isoliert erscheinen, hinsichtlich einer älteren Nibelungentradition nichts auszusagen, denn auch die Namen des Nibelungenlieds gehören zum reichen frühmittelalterlichen Personennamenschatz« (S. 18). Angesichts des Gewichts, das Heinzle der angeblichen Hausüberlieferung bei der Tradierung des Stoffs zuspricht, erscheint mir folgende Forderung berechtigt:

5. Der Versuch, snibelungische Haustradition wahrscheinlich zu machen, bedarf 'starker Argumente - ein Bündel sschwacher Indizien genügt nicht. Die Hypothese wäre dann akzeptabel, wenn eine sicher erschlossene Kleinfamilie namhaft gemacht werden kann, die über mehrere Generationen hinweg ihre Kinder in signifikantem Ausmaß mit verschiedenen Namen aus dem Nibelungenstoff benannt hat.

Bereits jetzt möchte ich vorwegnehmen, daß keines der von der Forschung vorgestellten Zeugnisse diesem hohen Anspruch genügt. Man kann natürlich die Hürde auch tiefer legen, doch das Risiko, daß man in diesem Fall ein Opfer des eigenen Wunschdenkens wird, erscheint mir zu hoch. Eine Indizienkette ist nur so stark wie ihr stärkstes Glied: Mit einer Fülle vager Anhaltspunkte kann man auch `beweisen , daß die Götter Astronauten waren ...

Bei der Vorbereitung seines Passauer Vortrags hat Störmer sich einen Ausdruck der in Freiburg mit EDV erstellten Prosopographie frühmittelalterlichen Personen für die Namen Nibulung, Gernot, Giselher, Sigur, Krimhilt besorgt. Die Auswertung, so Störmer, habe ihm gezeigt, daß diese Nibelungennamen in den außerbayerischen Quellen »fast in der Regel sehr isoliert stehen und daher wenig aussagekräftig sind « (S. 7). Die weitreichenden genealogischen Konstruktionen von Wenskus ${ }^{28}$ konnten also, so ist daraus zu folgern, von Störmer anhand dieses Materials nicht bestätigt werden. Eine eigene Nachfrage bei der Duisburger Personendatenbank erbrachte ebenfalls keinerlei signifikante Zusammenhänge. Sind etwa in einem Eintrag eines Verbrüderungsbuches zwei Träger verschiedener Heldennamen gemeinsam vertreten, so ist der Annahme einer zufälligen Kombination der Vorzug vor allen anderen Hypothesen zu geben. ${ }^{29}$

Wenn heroische Stoffe tatsächlich nur von einer relativ kleinen aristokratischen Schicht getragen wurden, so liegt die Frage nahe, ob in der sogenannten ,Reichsaristokratie, also der um die karolingischen Herrscher gruppierten

28 Eine Übernahme der personengeschichtlichen Hypothesen von Wenskus verbietet sich aufgrund der überzeugenden Kritik Eckhard Freises an seiner Identifizierungspraxis, vgl. E. Freise, Studien zum Einzugsbereich der Klostergemeinschaft von Fulda, in: K. Schmid (Hrsg.), Die Klostergemeinschaft von Fulda im früheren Mittelalter, München 1978 (Münstersche Mittelalter-Schriften 8/2.3), Bd. 2.3, S. 1003-1269, hier S. 1049 Anm. 296 ("Ausfluß beliebiger Namenkombinatorik«). Vgl. jetzt auch die deutlichen Worte von Althoff [Anm. 11], S. 40.

29 Herrn Prof.Dr.D. Geuenich, Duisburg, und seinem Mitarbeiter, Herrn Schreml, bin ich für eine Überlassung eines umfangreichen Ausdrucks sehr zu Dank verpflichtet. Gefragt war nach dem gemeinsamen Vorkommen der Namen Brunhild, Gernot, Giselher, Grimhild, Gunther, Hagen, Nibelung, Sigfrid, Sigmund, Sigur 
kleinen Elite, nibelungisches Bewußtsein greifbar wird. Wenn man den Begriff der >Reichsaristokraties, der im übrigen umstritten ist, nicht unzulässig ausweitet, kann man die Frage aufgrund der Lektüre der nicht wenigen Arbeiten der historischen Forschung zu dieser Gruppe ohne weiteres verneinen. Auch Störmer hat eine solche These nicht vertreten. Bereits jetzt erscheint fraglich, ob eine Handvoll westbayerischer Provinzmagnaten tatsächlich den Beweis dafür liefern kann, daß adeliger Hausüberlieferung bei der Tradierung heroischer Stoffe entscheidende Bedeutung zukam. Ich formuliere diesen negativen Befund in These 6:

6. Weder im Namensgut der, Reichsaristokratier noch in dem des, grundherrschaftlichen Adelsi außerhalb Bayerns sind glaubhafte Hinweise auf die Verwendung heroischer Namen als Haustradition zu konstatieren.

Als grundherrschaftlicher Adel mag jene Schicht vornehmer Freier bezeichnet werden, die über ausgedehnten Grundbesitz in mehreren Orten sowie zahlreiche Unfreie - Manzipien - verfügten und als Stifter von Eigenkirchen in Erscheinung traten. Dabei sollte man jedoch nicht übersehen, daß der »Übergang zwischen dem großen freien Bauern, dem kleinen Grundherrn und dem Adel [...] noch fließend « war. ${ }^{30}$

Ich diskutiere im folgenden vor allem die 1987 vorgelegte Arbeit Störmers. Starke Argumente werden für den Kernbereich der Beweisführung überhaupt nicht beigebracht, vielmehr müssen eine Reihe vager Indizien die Beweislast tragen. Zirkulär mutet die Argumentation in den am Ende formulierten Ergebnissen an: Nachdem Störmer festgestellt hat, sowohl in Bayern als auch im Wormsgau habe eine Familie gefunden werden können, in der zwei oder mehr Personennamen aus dem Nibelungensagenkreis zusammentreffen, schließt er, der Nibelungenstoff sei schon in der frühen Karolingerzeit bekannt gewesen. In Wirklichkeit ist dies überhaupt die Prämisse, die der Auswahl bestimmter sondernamen aus dem großen althochdeutschen Namensgut und damit auch der Konstruktion nibelungischer Personenverbände zugrunde liegt. Um etwa feststellen zu können, daß in einer Familie die Namen Sigifrid und Gunther als Heldennamen vergeben wurden, muß man die Bekanntheit heroischer Stoffe voraussetzen.

Geht man davon aus, daß sich das Personal des Nibelungenliedes erst im Zuge langer Tradierungsprozesse herausgebildet hat, so wird man zwischen den einzelnen Personennamen des Textes durchaus differenzieren müssen. Wenskus erörtert das Problem der Haupt- und Nebendarsteller ebensowenig wie die Frage, ob auch die iSchurken der Heldensage als Namenspatrone das aristokratische Prestige erhöhten. Für den Namen Hildebrand hat Störmer eine entsprechende Erwägung angestellt. Da ein Blutsverwandter des bayerischen Herzogs Tassilo Hildebrand heißt, weshalb »zweifellos« - so Strömer

und Sintarfizzilo. Bei den Quellen handelte es sich vornehmlich um Memorialquellen, d.h. die bayerischen Urkunden sind noch nicht erfaßt. Eine Kombination von drei Namen konnte nirgends festgestellt werden.

30 H.K. Schulze, Vom Reich der Franken zum Land der Deutschen: Merowinger und Karolinger, Berlin 1987, S. 246. 
noch 1987 - Nibelungenbezüge der Agilolfinger bestünden ${ }^{31}$, könnte man sich fragen, weshalb der Name des Sohnes Hadubrant in den bayerischen Quellen nicht erscheint. Störmers Antwort, der Name des Sohnes, der den Vater erschlägt, sei als "nicht traditionskräftig " betrachtet worden ${ }^{32}$, erscheint als ad-hoc-Hypothese, um die drohende Falsifikation der Theorie abzubiegen. Im übrigen haben die mit großem Aufwand wiederholt unternommenen Versuche, die Personennamen des Hildebrandliedes mit historischen Personen in Verbindung zu bringen, meines Wissens keinen nachhaltigen Erfolg gehab ${ }^{33}$.

Wenskus legt besonderen Wert auf die Namensvariation. Ein fiktives Beispiel: Aus den Brüdern Sigiher, Guntbert und Fridrich konnten in der Kindergeneration Sigifrid und Gunther werden. Mir erscheint diese Beobachtung durchaus wichtig. Wenn die Namensvariation in der Karolingerzeit relativ häufig vorkam - die neuere historische Forschung scheint dies zu belegen ${ }^{34}$ so rechtfertigt dies meines Erachtens die Forderung, daß in Familien mit Nibelungennamen hinsichtlich dieser Namen die Nachbenennung und nicht die Namensvariation über mehrere Generationen hinweg nachgewiesen werden müßte, um auszuschließen, daß die Nibelungennamen zufällig aus der Namensvariation hervorgegangen sind. Denn die sehr häufigen Anfangsglieder Gund-, Sig- oder Gisel- hat noch niemand exklusiv für die Heldensage beanspruchen wollen. Erst wenn eine Familie gefunden wird, in der mindestens zwei Heldennamen über mehrere Generationen hinweg eben nicht verändert werden, will ich eingestehen, daß die zu prüfende Hypothese plausibler geworden ist. Um einen Zufall kann es sich freilich auch dann noch handeln denn: ein Zeuge ist kein Zeuge. Angesichts der dürftigen Quellenlage und der Bemühungen der germanistischen Forschung seit den Brüdern Grimm um Heldennamen halte ich das Auffinden einer solchen Familie freilich für äuBerst unwahrscheinlich.

Störmers Quellen sind die Freisinger Traditionen des 8. und 9. Jahrhunderts, äußerst reizvolle und aussagefähige, aber auch recht schwierige Dokumente. ${ }^{35}$ Die von Störmer und anderen betriebene Konstruktion von Adelsfamilien aufgrund des zufällig erhalten gebliebenen Freisinger Materials ist wiederholt erheblich kritisiert worden. In der Rezension von Störmers 1972 veröffentlichtem Buch über bayerische Adelsgruppen, der personengeschichtlichen Grundlage der Monographie über den `Frühen Adek, bemerkte Rudolf

31 Störmer [Anm. 6], S. 10.

32 Störmer [Anm. 4], Bd. 2, S. 491.

33 Vgl. auch D. Geuenich, Die Personennamen der Klostergemeinschaft von Fulda im früheren Mittelalter, München 1976 (Münstersche Mittelalter-Schriften 5), S. 228f.; E. Stutz, Hadebrant und Alebrant, Beiträge zur Namenforschung NF 19 (1984), S. $261-274$.

34 Vgl. zuletzt H.-W. Goetz, Zur Namengebung bäuerlicher Schichten im Frühmittelalter. Untersuchungen und Berechnungen anhand des Polyptichons von Saint-Germain-des-Prés, Francia 15 (1987), S. 852-877, hier S. 861.

35 Die Traditionen des Hochstifts Freising, hrsg. v. Th. Bitterauf, Bd. 1-2, München 1905-1909 (Quellen und Erörterungen zur bayerischen und deutschen Geschichte NF 4-5). 
Schieffer, das Buch offenbare auch die Grenzen jener Forschungsrichtung, der es um die verwandtschaftliche Verflechtung überregionaler Adelsgruppen gehe. Dies gelte insbesondere für den von Störmer weithin betriebenen reinen Namensvergleich. Störmers auf die Namensindices der einschlägigen Urkundenbücher gestütztes Kombinationsgeschick lasse sich "selbst von so verbreiteten frühmittelalterlichen Namen wie Gunther und Hugbert nicht abschrekken « ${ }^{36}$ Gunther ist im übrigen, dies gilt es festzuhalten, der wohl wichtigste von Störmers Nibelungennamen.

Mehrfach hat man darauf aufmerksam gemacht, daß die Behauptung, mehrere westbayerische Klöster seien von den sogenannten Huosi gegründet worden, sich wesentlich auf eine Identifizierung zweier Personen des Namens Eio stützt, die von den Quellen her sogar als eher unwahrscheinlich erwiesen werden kann. ${ }^{37}$ Störmer verortet die Nibelungennamen im Huosi-Clan, ohne daß deutlich wird, daß dieser Huosi-Clan eine durchaus angefochtene Konstruktion ist, die bei den einzelnen Autoren erheblich differiert. ${ }^{38}$. Wenn man einem Strang, der einschlägig tätigen Forschung glaubt und die Gründungsfamilien der westbayerischen Klöster für die Huosi in Anspruch nimmt, stellt man erstaunt fest, daß die angeblich so prestigesteigernden Heldennamen unter diesen Gründungsfamilien eben nicht vertreten sind. ${ }^{39}$

Wir kennen dank einer Freisinger Tradition aus dem Jahr 791 einige Angehörige der äußerst vornehmen genealogia der Huosi namentlich (Nr. 142). Zu ihnen gehört ein Nibelung, der ohne zusätzliche Argumente mit dem Zeugen einer Tradition von 802 gleichgesetzt wird (Nr. 182). Die Schenker von 802, die Priester Wenilo und Helmker, werden von Störmer als Huosier bezeichnet, obwohl es dafür keinen Beleg gibt. Seine Aussage, daß sich nicht »weniger als drei Nibelungennamensträger « unter den Zeugen vorfinden, ist in ihrem Beweiswert doch etwas zu relativieren. 15 Zeugen werden bei der Schenkung

36 R. Schieffer, Blätter für deutsche Landesgeschichte 110 (1974), S. 650.

37 Schieffer [Anm. 36], S. 650; A. Kraus, Die Grenzen der kartographischen Methode, Blätter für deutsche Landesgeschichte 113 (1977), S. 33-43, hier S. 37 Anm. 26; L. Holzfurtner, Untersuchungen zur Namengebung im frühen Mittelalter nach bayerischen Quellen des achten und neunten Jahrhunderts, Zeitschrift für bayerische Landesgeschichte 45 (1982), S. 3-21, hier S. 20f. Auch Störmer lehnt die These der Huosi-Klöster ab, vgl. W. Störmer, Adelsgruppen im früh- und hochmittelalterlichen Bayern, München 1972 (Studien zur bayerischen Verfassungs- und Sozialgeschichte 4), S. 99, 101.

38 Die kritischen Einwände von L. Holzfurtner, >Pagus Huosi< und Huosigau. Untersuchungen zur Gaulandschaft im westlichen Oberbayern, in: A. Kraus (Hrsg.), Land und Reich, Stamm und Nationo, Bd. 1, München 1984 (Schriftenreihe zur bayerischen Landesgeschichte 78), S. 287-304, hier S. 294, haben den bayerischen Freundeskreis der Huosi nicht beirren können: vgl. G. Mayr, Ebersberg. Gericht Schwaben, München 1989 (Historischer Atlas von Bayern. Altbayern 18), S. 62; W. Störmer, Huosi, in: Lexikon des Mittelalters, Bd. 5, Lief, 2, 1990, Sp. 229-230.

39 Dies geht etwa aus der Arbeit von Maria Neumann hervor, die den Kreis der sHuosi< wohl am weitesten faßte: M. Neumann, Die bairische Volksordnung zur Karolingerzeit aufgrund genealogischer Untersuchungen, Diss. masch. Erlangen 1947. Das von ihr entworfene Bild der bairischen Sozialgeschichte erscheint mir differenzierter und überzeugender als das bei Störmer gebotene. 
aufgeführt, doch nur Nipulunc und Cundheri stehen als zweiter und dritter Zeuge nebeneinander, Sigifrid erscheint ganz am Ende der Zeugenreihe.

Der Name Nibelung tritt in den Freisinger Traditionen genau dreimal auf. Wenn man annimmt, daß es sich jedesmal um den Huosier handelt, so ist er nur ein einziges Mal mit Personen mit Nibelungennamen vergesellschaftet. Auch die Beziehung Nibelungs zu den angeblichen Huosi Wenilo und Helmker $^{40}$ trägt nicht. Zeugen waren, mag dies von der genealogisch-besitzgeschichtlichen Forschung auch meist geleugnet werden, eben nicht nur Verwandte, sondern oft genug Nachbarn oder angesehene Leute an dem Ort der Schenkung. ${ }^{41}$ Plausibel machen läßt sich dies durch einen Blick auf die Bestätigung der Schenkung von 802 durch Wenilo im Jahr 836 (Nr. 612). Weder erscheint in dieser Tradition der Träger eines Nibelungennamens noch auch nur ein einziger der Zeugen von 802.

Halten wir fest: Wie auch immer die Huosi zu beschreiben sein mögen, ob als Adelsfamilie, als Clan oder als völkerwanderungszeitlicher Stammessplitter - konkrete Anhaltspunkte, daß diese Gruppe ein nibelungisches Hausbewußtsein gepflegt hat, lassen sich nicht ausmachen. Natürlich kann man behaupten, daß alle westbayerischen Freien in den Freisinger Traditionen mit den Huosi verwandt waren. >Irgendwier stimmt das immer. Doch wenn man an meiner These 5, die starke Argumente fordert, festhält, braucht von den Huosi und somit von der Spitzengruppe der bayerischen Oberschicht fortan nicht mehr die Rede zu sein.

Es wäre allerdings immer noch interessant, wenn man eine Familie ausfinden machen könnte, in der mehrere Nibelungennamen vertreten wären. Ein besonders eindrucksvolles Beispiel aus dem Jahr 774 hat Weddige aus den Lorscher Traditionen bekanntgemacht: Ein Gundhar mit Frau Willihilt hat als Kinder Waldhar und Brunihilt (S. 74). Ich denke, auch dabei kann es sich um Zufall handeln. Interessant ist jedenfalls, daß der Beleg nicht besonders geeignet ist, aristokratisches Adelsheil qua Heldennamen zu bezeugen. Die genannten Personen sind nämlich Hörige, also Unfreie.

Bei der Suche nach Familien wird man in Störmers Aufsatz kaum fündig. Er stützt sich bei der Annahme von Verwandtschaftsbeziehungen auf wenig akzeptable Indizien wie patronymische Ortsnamen, räumliche Nähe von Orten und gemeinsame Nennung in Zeugenlisten. In Kottgeisering, das mit dem Kysalheringa einer Freisinger Tradition gleichgesetzt wird, zeugt 829 ein Mann »mit der nordischen Form des Siegfried: Sigur« (S. 8). Wann der Giselher, der Kysalheringa den Namen gegeben hat, gelebt hat, ist durchaus unklar, der Bezug also kaum zu verwerten. Doch Störmer fährt fort: »Bezeichnenderweise« liege dieser Ort unmittelbar bei Unteralting, wo ca. 805-809 ein

40 Gertrud Diepolder, die sich selbst als »nachgerade [...] huosischeue Autorin « apostrophiert (S. 172), diskutiert Identifizierungsmöglichkeiten für Personen des Namens Helmker, vgl. G. Diepolder, Schäftlarn: Nachlese in den Traditionen der Gründerzeit, in: I. Eberl/W. Hartung/J. Jahn (Hrsg.), Früh- und hochmittelalterlicher Adel in Schwaben und Bayern, Sigmaringendorf 1988 (Regio 1), S. 161-188, hier S. 171-173.

41 Vgl. Althoff 1990 [Anm. 11], S. 24. 
Grundherr Kysalheri begegnet. Nun, der Ausstellungsort der Tradition lautet Alamunting $a$, und ob damit Ober- oder Unteralting gemeint ist, ist, wie in den Freisinger Traditionen leider recht häufig, durchaus nicht eindeutig. Ein Blick auf den Atlas macht deutlich, daß Ober- und Unteralting alles andere als unmittelbar benachbart sind.

Der Grundherr Giselher von Ober- oder Unteralting erscheint in der Tradition Nr. 200c zusammen mit einem Sigifrid. Giselher ist Spitzenzeuge, Sigifrid der zwölfte von 18 Zeugen. Die Zeugenlisten der insgesamt sieben Rechtsgeschäfte, die in der Tradition Nr. 200 zusammengefaßt sind und die sich über die Jahre 804 bis 810 erstreckten, weisen durchaus signifikante Überschneidungen auf. Sigifrid wird freilich nur in einer einzigen dieser Rechtshandlungen, der Tradition des Deotpert - nicht etwa des Giselher - erwähnt. Angesichts dieses Befundes eine engere Beziehung zwischen Giselher und Sigifrid herzustellen, wie Störmer dies tut, erscheint mir nicht angebracht.

Der Eindruck eines 'Nestes` von Nibelungennamen drängt sich bei unbefangener Lektüre der Freisinger Traditionen durchaus nicht auf. Wenn man das Personenregister auf Nibelungennamen durchsieht, begegnet man zahlreichen Nennungen, von denen Störmer nur die auf einen bestimmten Raum und die Freien bezogenen verwertet und mit einer Fülle ineinandergreifender Hypothesen in einen verwandtschaftlichen Zusammenhang bringen möchte. $\mathrm{Da}$ es sich bei den Zeugen oder Grundbesitzern um Adelige handelt, wird lediglich vorausgesetzt, denn die mitunter auftretende Quellenbezeichnung nobilis benennt in den Freisinger Traditionen den (voll)freien Mann. ${ }^{42}$

Bezeichnend erscheint mir die Besprechung der Tradition Nr. 225 durch Störmer. Ein Mann namens Nahuni schenkte 806 für sich, seinen Vater Deotuni, seine Mutter Hrodni und seine Töchter Chrimhilt und Kysalni Besitz in Maisach. Die neun Zeugen tragen, was Störmer übergeht, keine Nibelungennamen. Wer dieser Nahuni war, ist unbekannt. Vielleicht war es ein kleiner bäuerlich lebender Freier, dessen einzige historische Bedeutung darin besteht, seiner Tochter den Namen Kriemhilt gegeben zu haben, der nur dieses eine Mal in den Freisinger Traditionen auftritt. Nahuni oder seine Familie läßt sich nicht mit anderen Namensträgern in der Freisinger Überlieferung in Verbindung bringen.

Weshalb Störmer meint, Hrodni und Kysalni seien ausgesprochen nordische Namensformen, ist mir unverständlich, zumal das Endglied -ni (bzw. lemmatisiert ıneui<) im althochdeutschen Namensschatz häufig vertreten ist und auch das Stammglied Hrod- weit verbreitet war. Gleiches gilt für den Namen Sigur, der von Störmer unrichtig als nordische Form des Heldennamens Siegfried identifiziert und als Indiz für »frühe nordische Beziehungen des bayerischen `Nibelungenkreises« (S. 15) in Anspruch genommen wird -

42 Vgl. schon Bitterauf [Anm. 35], Bd. 1, S. LXXVIII; J. Sturm, Die Anfänge des Hauses Preising, München 1931, Neudruck Aalen 1974 (Schriftenreihe zur bayerischen Landesgeschichte 8), S. 238; Neumann [Anm. 39], S. 273-275; vgl. allgemein auch J. Schmitt, Untersuchungen zu den Liberi homines der Karolingerzeit, Frankfurt a.M./Bern 1977 (EHS Hochschulschriften II, 83), S. 82f., 88; Schneider IX [Anm. 22], S. 336-347. 
zusammen mit Hrodni und Sintarfizzilo. Jedenfalls geben die angeblich nordischen Namen Störmer Gelegenheit, Wenskus Thesen über gentile Traditionskerne zustimmend zu referieren, obwohl deren empirisches Fundament mehr als fraglich ist.

Die von der jüngeren Forschung zunehmend skeptisch gesehenen überregionalen Bezüge der von Wenskus und anderen konstruierten Adelsgruppen werden auch bei der Besprechung einer Schenkung eines Grafen Gunther um 750 in Türkenfeld - Duringveld vorausgesetzt. Die Ableitung des Ortsnamens vom Volksnamen der Thüringer in Verbindung mit Wenskus' Hinweis auf thüringische Nibelungenbeziehungen führt zur Behauptung Störmers, der westbayerische Raum sei »offensichtlich" eine »ethnische Mischzone« mit einer möglicherweise polyethnischen Herrenschicht. Darauf verwiesen "ganz besonders die nordischen Namen, wie etwa Sigur« (S. 13).

Bei der aus den Lorscher Traditionen erschlossenen Nibelungensippe im Wormsgau handelt es sich ebenfalls um eine reine Konstruktion, die nicht als gesicherte Familie gelten kann. Daß in dem von Gottfried Mayr erstellten äußerst hypothetischen Stemma die Namen Siglind und Gundheri vorkommen, bezeichnenderweise nach Störmers Eingeständnis "eher am Rande" (S. 14), beweist im Grunde gar nichts. ${ }^{43}$ Auch der angebliche Nachweis einer Verbindung zwischen bayerischen und Wormsgauer Namensträgern kann nicht als erbracht gelten.

Alle von Störmer im westbayerischen Raum behaupteten verwandtschaftlichen Bezüge zwischen Personen mit Nibelungennamen sind durchwegs hypothetisch. Bis zum Jahr 850 sind über 700 Freisinger Traditionen überliefert. Die aus ihnen herangezogenen wenigen Zeugnisse sind keinesfalls $>$ dicht in dem Sinne, daß sie sich auf eine räumlich und zeitlich eng begrenzte soziale Gruppe beziehen. Ich denke, man wird deshalb die nächste These verstehen können:

7. Störmers Versuch, wenigstens im westbayerischen Raum mibelungische Haustraditione im Umkreis der sgenealogiar der Huosi aufzuweisen, scheitert daran, daß den verwerteten Freisinger Traditionsnotizen keine handfesten Anhaltspunkte für eine verwandtschaftliche Beziehung der Personen mit Nibelungen-Namen zu entnehmen sind.

Seit dem Anfang unseres Jahrhunderts bemüht sich die bayerische Forschung um einen vornehmen Herrn namens Piligrim, der von 814 bis 853 in zahlreichen Freisinger Traditionsurkunden begegnen soll und vor allem in Allershausen die Grundbesitzer faßbar ist. ${ }^{44}$ Vorausgesetzt wurde stets, daß es sich immer um denselben Piligrim handelt. In gleicher Weise hypothetisch ist die für seine angebliche Familie erstellte, von Störmer übernommene Stammtafel. Daß Piligrim einen Bruder Oadalker hatte, ist eine Annahme, die immerhin

43 G. Mayr, Studien zum Adel im frühmittelalterlichen Bayern, München 1974 (Studien zur bayerischen Verfassungs- und Sozialgeschichte 5), S. 80.

44 Ich nenne nur Sturm [Anm. 42], S. 219f.; Neumann [Anm. 39], S. 20-34; M. Mitterauer, Karolingische Markgrafen im Südosten. Fränkische Reichsaristokratie und bayerischer Stammesadel im österreichischen Raum, Wien 1963 (Archiv für österreichische Geschichte 123), S. 229-235. Die herrschende Meinung formuliert Mayr 
mit zusätzlichen Argumenten gestützt werden kann, während die Vermutung, $\mathrm{da} ß$ ein anderer Bruder Haduperht hieß, lediglich auf den Umstand zurückgeht, daß der Vater dieses Haduperht den gleichen Namen trug wie auch Piligrims Vater. Doch selbst wenn man das Stemma akzeptiert, vermißt man einen Nibelungennamen. Das Brückenglied stellen die beiden Namen Oadalker (der erschlossene Bruder) und Reginpert (der gesicherte Sohn Piligrims) dar, die mit den Huosiern des Namens in Verbindung gebracht werden. Außer der Namensgleichheit gibt es dafür keine weiteren Anhaltspunkte. Wenn die 'Huosi-Connection der Nibelungen, wie oben behauptet, nicht akzeptabel ist, so gilt dies noch viel mehr für Störmers Behauptung, Bischof Pilgrim von Passau (971-991) stamme in mehr als einer Linie von Pilgrim von Allershausen $\mathrm{ab}$, dieser habe ihm nibelungisches Abstammungsbewußtsein vermittelt und es gebe daher kein »echtes Argument«, so Störmer 1974, das die von der 'Klage behauptete lateinische Niederschrift der Nibelungenüberlieferung durch Bischof Pilgrim von Passau widerlegen könne (S. 66).

In dem Aufsatz von 1974 sichtet Störmer zunächst alle bayerischen Namensträger Piligrim von 800 bis 1000 , die er auf zehn Personen bezieht. Diese werden ohne Begründung als adelig bezeichnet. $\mathrm{Da}$ es nur zehn sind, gilt ihm als »Bewußtsein einer engen Familientradition « (S. 64). Daß alle miteinander verwandt sind, ist jedoch von Störmer keinesfalls erwiesen worden. Das nächste Argument weist den Passauer Bischof dieser Piligrim-Gruppe zu. Störmer wörtlich: »Daß dem so ist, scheint mir die Nibelungen-Überlieferung zu beweisen« (ebd.). Wenn aber die Nibelungenüberlieferung Bischof Pilgrim mit den anderen Trägern dieses Namens in Verbindung bringen soll, kann diese Verbindung nicht gleichzeitig als der Nachweis der tatsächlichen Bezüge der historischen Person Pilgrim von Passau zur Nibelungenüberlieferung betrachtet werden.

Im übrigen sind die von der Forschung hergestellten Verbindungen zwischen Piligrim von Allershausen und dem Bischof sehr hypothetisch. Sowohl die verwandtschaftlichen Beziehungen des Allershauseners als auch die des Bischofs zu den Aribonen und Luitpoldingern sind alles andere als gesichert. Um so mehr gilt das für die direkte Verbindung beider Personen - Hypothesen, wohin man blickt. Angesichts der weitreichenden Bedeutung, die dem Nachweis tatsächlicher nibelungischer Hausüberlieferung im Umkreis Bischof Pilgrims von Passau für die Heldensagenforschung zugemessen wird, halte ich eine vermeintlich hyperskeptischer Haltung für weniger risikoreich und vertrete daher These 8:

8. Ebenfalls abzulehnen ist die Behauptung, eine vor allem in Allershausen begüterte Person namens Pilgrim aus der ersten Hälfte des 9. Jahrhundert sei ein Vorfahr Bischof Pilgrims von Passau (971-991) gewesen und habe diesem snibelungische Haustradition vermitteln können. Die von der, Klager behauptete Mitwirkung des Passauer Bischofs an der Genese des Epos hat durch Störmers Hypothese nichts an Wahrscheinlichkeit gewonnen.

[Anm. 38], S. 100 Anm. 830: »Piligrim war ein zu den Huosi gehörender Stammvater der Aribonen«. 
Folgen wir noch einmal dem Gang der Erörterung. Die ersten vier Thesen skizzierten den methodischen Rahmen der historischen Fragestellung. These 5 gab dann eine ohne weiteres empirisch überprüfbare Bedingung für das Vorliegen eines überzeugenden Zusammenhangs an. Die Hürde wurde angesichts der weitreichenden Bedeutung, die dem Resultat zukommen soll, vermeintlich hoch angesetzt. Gefordert wurden jedoch nicht hundert, nicht zwanzig, nicht zehn oder fünf Familien, sondern genau eine Familie. Erfüllen die eher gut bekannten Geschlechter der karolingischen Elite das gewählte Kriterium? Das war zu verneinen. Da die spekulative Konstruktion nibelungischer Traditionsverbände durch Wenskus selbst von Störmer nicht aufrechterhalten wurden, waren auch die Familien des außerbayerischen grundherrschaftlichen Oberschicht auszuschließen. War nibelungische Tradition dann wenigstens in der Spitzengruppe der bayerischen Oberschicht, bei den Huosi, präsent? Auch diese Aussage konnte nicht bejaht werden. Die von Störmer aus den Freisinger Traditionen namhaft gemachten Personen mit Nibelungennamen können weder sicher als Adelige noch als Verwandte bezeichnet werden. Bei unbefangener Betrachtung stellen sich diese Nennungen als das Produkt einer spezifischen Optik heraus, nämlich der des Forschers, der nach Heldennamen Ausschau hält.

Dieser Befund ist nun noch anhand der erzählenden Quellen zu überprüfen. Adelige Abstammungserzählungen, in meiner Terminologie sgenealogische Herkommen ${ }^{45}$, sind nur in sehr geringer Zahl aus dem Mittelalter überliefert. ${ }^{46}$ Genealogische Herkommen aus dem Bereich der Heldensage begegnen mit einer Ausnahme im 12. Jahrhundert (Wiprecht von Groitzsch), die freilich auch als Produkt klösterlicher Überlegungen verdächtigt werden kann, jedoch erst in der zweiten Hälfte des 15. Jahrhunderts, meist sind es unverkennbar gelehrte Kombinationen. ${ }^{47}$

Die mittelalterlichen Herkommensüberlieferungen des Adels wurden, soweit erkennbar, von ausgesprochenen `Moden bestimmt. Früh- und hochmittelalterliche Familien leiteten sich gern vom heiligen Arnulf oder anderen Angehörigen des Karolingerhauses, nicht zuletzt von Karl dem Großen ab. Sächsische Adelsfamilien bevorzugten seinen Gegner Widukind. Alberich von Troisfontaine bemerkte dazu im 13. Jahrhundert, der ganze Adel Europas stamme von Widukind ab. ${ }^{48}$ Im Spätmittelalter dominierend war die Herleitung des Adels von stadtrömischen Familien. ${ }^{49}$ Gegen dieses Römer-Herkom-

45 Graf [Anm. 12].

46 Vgl. ausführlich Althoff 1990 [Anm. 11], S. 67-77; ders. 1988 [Anm. 11].

$47 \mathrm{Vgl.} \mathrm{K}$. Graf, Heroisches Herkommen. Überlegungen zum Begriff der »historischen Überlieferung« am Beispiel heroischer Traditionen (erscheint in: Petzoldt/S. de Rachewiltz [Hrsg.], Sage und Geschichte).

48 G. Althoff, Genealogische und andere Fiktionen in mittelalterlicher Historiographie, in: Fälschungen im Mittelalter. Teil I, Hannover 1988 (MGH Schriften 33, I), S. 417-441, hier S. 421.

49 Vgl. etwa K. Graf, Exemplarische Geschichten. Thomas Lirers "Schwäbische Chronik« und die "Gmünder Kaiserchronik«, München 1987 (Forschungen zur Geschichte der älteren deutschen Literatur 7), S. $122 \mathrm{f}$. 
men zogen die Humanisten zu Felde, die Ableitungen von germanischen Helden präferierten. Natürlich gab es daneben auch `exklusive` Herkommen, etwa die Schwanrittersage ${ }^{s_{0}}$, die nur wenigen Familien zukamen. Entscheidend scheint mir aber zu sein, daß Ableitungen vom Personal der Heldensage mit Sicherheit nicht weit verbreitet waren, da sie sonst häufiger bezeugt sein müßten.

Dagegen ist bekannt, daß sich nordische Könige von Siegfried herleiteten. Wenskus mißt diesem Befund besonderes Gewicht zu. ${ }^{51}$ Der Blick auf die quellenmäßig bezeugten Abstammungsfiktionen mittelalterlicher Adelsfamilien vermochte gleichsam die Gegenprobe zu machen und zu verdeutlichen, daß ein der nordischen Praxis vergleichbares Phänomen im Adel des deutschsprachigen Raums nicht nachweisbar ist.

Die Conclusio kann angesichts der vorgetragenen Argumente nicht überraschen:

Alle bisherigen Bemühungen, 'Heldennamen als Bestandteil des Selbstverständnisses des sfühen Adels $\mathbf{z}$ erweisen, sind aufgrund ihres allzu hypothetischen Charakters nicht akzeptabel. Angesichts der Quellenlage ist mit wesentlich neuen Argumenten nicht zu rechnen. Die auf den ersten Blick faszinierende Möglichkeit, die Tradierung heroischer Stoffe mit aus der Namengebung erschlossener sadeliger Hausüberlieferung zu korrelieren, muß somit wohl als ,Sackgasser betrachtet werden.

Was bedeutet dieses negative Ergebnis für die literarische Interessenbildung, für die Produktion und Rezeption heroischer Texte? Einer sozialhistorisch orientierten Funktionsgeschichte der Literatur wird damit keineswegs der Boden entzogen..$^{52}$ Es soll nicht einmal ausgeschlossen werden, daß etliche der erwähnten Träger von Heldennamen tatsächlich nach Vorbildern der Heldensage benannt wurden. Träfe diese - derzeit nicht bewiesene - Annahme zu, so wäre zu fragen, ob die Erklärung als archaische Hausüberlieferung in einem solchen Fall überhaupt zwingend oder auch nur naheliegend wäre. Denn nicht nur das Herkommen der eigenen Familie, sondern auch der exemplarische Gehalt des Stoffes konnte sich als Anknüpfungspunkt anbieten. Nach Ordericus Vitalis hat ein Normannenherzog seinem Sohn den Namen Boemund nach einer in convivio ioculari vorgetragenen Geschichte von einem Riesen Buamund gegeben. ${ }^{53}$ Wäre ein Heldenname, der dem auch sonst gebräuchlichen Namensgut entstammt, >vererbt« worden, so ließe sich übrigens nicht ohne weiteres entscheiden, ob diese $>$ Hausüberlieferung ( ihren ursprünglichen heroischen Sinn bewahrt hätte.

50 Vgl. Graf [Anm. 12].

51 Wenskus 1976 [Anm. 3], S. 479f.

52 Die folgenden Bemerkungen sind besonders dem Abschnitt »Funktionen der Heldensage« bei Haubrichs [Anm. 25], S. 134-144 verpflichtet, führen aber auch die bei Graf [Anm. 47] entwickelten Positionen weiter.

53 Vgl. W. Grimm, Die deutsche Heldensage, 3. Aufl., hrsg. v. R. Steig, Gütersloh 1889 , S. 461. 
Das auf den ersten Blick einleuchtende Argument, adelige Mäzene hätten mit der Förderung artifizieller Heldendichtung die Vergangenheit des eigenen Geschlechts preisen lassen, entzieht sich bekanntlich einer empirischen Überprüfung anhand der überlieferten Texte, da die sadelige Hausüberlieferung in ihnen keine eindeutig erkennbaren Spuren hinterlassen hat. Es erscheint mir daher an der Zeit, andere Formen der Aneignung heroischer Tradition als >Herkommen

$\mathrm{Zu}$ wenig berücksichtigt wird der mit dem Konzept der `Genealogie konkurrierende Gedanke der `Sukzession‘. Indem die einschlägigen Protagonisten der heroischen Stoffe als, Vorzeit-Herrscher, als nicht notwendigerweise mit ,Vorfahren im genealogischen Sinn identische >Vorgänger verstanden wurden, baute man sie ein in die eigene Geschichte, verstanden als Herkommen eines $>$ Herrscher-Amts ${ }^{55}$ Dieser höfische Modus der Rezeption ist mindestens ebensogut wie die Hypothese sadeliger Haustradition geeignet, die Genese anspruchsvoller literarischer Texte zu erklären.

Läßt man diese Texte am sozialen Mittelpunkt einer gentilen oder regionalen Gruppe entstanden sein, so können sie nicht nur als >gentiles Herkommen<, das die Helden in der Geschichte der eigenen Gemeinschaft wirken lie ${ }^{56}$, interpretiert werden, sondern auch, bezogen auf jene Texte, in denen (fremdes) ethnisches Schicksal zum Thema wird, als Medien der Kontingenzerfahrung. Daß ein solcher Versammlungsort oder sHof Mittelalter den Austragungsort von Kampfspielen abgab, die - wie die spätmittelalterlichen Rosengarten-Spiele - auf den Nachvollzug epischen Geschehens abzielten, kann zumindest nicht völlig ausgeschlossen werden.

Nimmt man die Institution >Hof als ।Sitz im Leben $<$ eines bestimmten Typs heroischer Dichtung in Anspruch ${ }^{57}$, sollte man sich nicht zu sehr an den Formen späterer dynastischer Repräsentation orientieren. Die genossenschaftliche Dimension des Höfischen darf nämlich nicht außer acht gelassen werden. Die bekannte Rolle Breisachs im Harelungen-Stoff kann möglicherweise mit einer Vermutung von Gerd Althoff verbunden werden, der in Breisach einen traditionellen Treffpunkt adeliger Verschwörer sieht. ${ }^{58}$ Zugegebenermaßen sehr spekulativ wäre zu erwägen, ob die wiederholten Bündnisse der Adeligen in Breisach nicht mit dem Vortrag heroischer Überlieferung verbunden gewesen sein könnten und Breisach über diese Vortragssituation Eingang in die Texte gefunden hätte.

$54 \mathrm{Zu}$ dieser Gegenüberstellung vgl. K. Graf, Thesen zur Verabschiedung des Begriffs der shistorischen Sage`, Fabula 29 (1988), S. 21-47, hier S. 46; Graf [Anm. 49], S. 21 f.; Graf [Anm. 12], S. 288, 290.

55 Vgl. dazu Haubrichs [Anm. 25], S. 140-144, doch würde ich den kontinuitätsstiftenden Gedanken der Sukzession stärker vom liturgischen Totengedenken, der memoria, trennen.

$56 \mathrm{Vgl}$. Haubrichs [Anm. 25], S. 139.

57 Vgl. Haubrichs [Anm. 25], S. 81-89, der das Höfische ganz dem Adel reserviert. Aber war etwa ein Bischofs-Hof tatsächlich ein reiner >Adelshof $s$ ?

58 G. Althoff, Breisach - ein Refugium für Rebellen im früheren Mittelalter, in: Archäologie und Geschichte des ersten Jahrtausends in Südwestdeutschland, Sigmaringen 1990 (Archäologie und Geschichte 1), S. 457-471. 
Angesprochen ist mit der Nennung von Breisach jedenfalls die Funktion heroischer Tradition als `Orts-Herkommen«, für die es im Gegensatz zur radeligen Hausüberlieferung unbestreitbare Zeugnisse gibt. ${ }^{59}$ Lokale und regionale Erzählgemeinschaften bezogen über Orts-Herkommen die Helden in die eigene Geschichte ein. Betonen möchte ich, daß keine vernünftigen Gründe dafür ersichtlich sind, die Träger heroischer Überlieferung ausschließlich in adeligen Kreisen zu suchen. Wenn das Ethos der Heldensage `Kriegerethos ist - weshalb sollten die frühmittelalterlichen `Bauernkrieger, die es nachweislich gegeben hat, nicht in ähnlicher Weise wie die Bauern der spätmittelalterlichen Schweiz dieses Ethos auch für sich als verbindlich erachtet haben?

Möglichkeiten, abseits der großen `Höfe heroische Stoffe kennenzulernen, waren allemal gegeben - auch wenn darüber aufgrund der Eigenart der Quellenüberlieferung so gut wie nichts verlautet. Bei solchen Gelegenheiten wird man eher seinfachere` Fassungen vorgetragen haben, und für die - durchaus nicht nebensächliche - breite Diffusion heroischer Stoffe im Medium der Mündlichkeit dürften nicht-exklusive Erzählsituationen wichtiger gewesen sein als die höfische Produktion und Rezeption des `klassischen Heldenlieds mit tragischem Konflikt. ${ }^{60}$ Meiner Ansicht nach konnten sich genealogische Herkommen in die nicht verschriftlichten (und daher auch textlich nicht überlieferten) Versionen heroischer Stoffe und Schemata sogar eher seinhängen` als in die ästhetisch anspruchsvolleren Texte. ${ }^{61}$

Verabschiedet man die Vorstellung, mit der Formel `Heldensage als adelige Hausüberlieferung sei der wichtigste Schlüssel zur literarischen Interessenbildung im Feld heroischer Stoffe gefunden, so ist zugleich die Chance eröffnet, zu einem differenzierteren Bild zu gelangen, das weder die Sozialstruktur der Epoche noch die Gattung zu monolithisch sieht. Die grobe Skizze einer eher spluralistisch s ausgeformten Alternative in meinen Schlußbemerkungen konnte und wollte jedoch die hierzu erforderliche literaturwissenschaftliche Diskussion nicht vorwegnehmen.

$59 \mathrm{Vgl}$. Haubrichs [Anm. 25], S. 139 (»lokale Anbindung«). Die spätmittelalterlichen Zeugnisse (Worms, Xanten u.a.) sind besprochen bei Graf [Anm. 47].

60 Vgl. dazu D. Hofmann, Vers und Prosa in der mündlich gepflegten mittelalterlichen Erzählkunst der germanischen Länder, FMSt 5 (1971), S. 135-175, hier S. 147f.

61 Die Funktion als genealogisches Herkommen ist denn auch vor allem bei den nur indirekt bezeugten 'Sageliedern` zu belegen, vgl. Haubrichs [Anm. 25], S. $170 \mathrm{f}$. 